\title{
Cardiovascular Regulation and Body Temperature: Evidence From a Nap vs. Sleep Deprivation Randomized Controlled Trial
}

\author{
J. SLOMKO ${ }^{1}$, M. ZAWADKA-KUNIKOWSKA ${ }^{1}$, J. J. KLAWE ${ }^{1}$, M. TAFIL-KLAWE ${ }^{2}$, \\ J. NEWTON ${ }^{3}$, P. ZALEWSKI ${ }^{1}$
}

${ }^{1}$ Department of Hygiene, Epidemiology and Ergonomics, Nicolaus Copernicus University in Torun, Ludwik Rydygier Collegium Medicum in Bydgoszcz, Bydgoszcz, Poland, ${ }^{2}$ Department of Human Physiology, Nicolaus Copernicus University in Torun, Ludwik Rydygier Collegium Medicum in Bydgoszcz, Bydgoszcz, Poland, ${ }^{3}$ Institute for Ageing and Health, The Medical School, Newcastle University, Newcastle-upon-Tyne, United Kingdom

Received September 8, 2017

Accepted May 14, 2018

\begin{abstract}
Summary
In this study we set out to understand is sleep fragmentation affects the cardiovascular regulation and circadian variability of core body temperature more or less than sleep deprivation. 50 healthy men (age $29.0 \pm 3.1$ years; BMI $24.3 \pm 2.1 \mathrm{~kg} / \mathrm{m}^{2}$ ) participated in a 3-day study that included one adaptative night and one experimental night involving randomization to: sleep deprivation (SD) and sleep fragmentation (SF). The evaluation included hemodynamic parameters, measures of the spectral analysis of heart rate and blood pressure variability, and the sensitivity of arterial baroreflex function. Core body temperature (CBT) was measured with a telemetric system. SF affects heart rate $(61.9 \pm 5.6$ vs. $56.2 \pm 7.6, p<0.01)$ and stroke index $(52.7 \pm 11.1$ vs. $59.8 \pm 12.2, \mathrm{p}<0.05)$ with significant changes in the activity of the ANS (LF-sBP: $6.0 \pm 5.3$ vs. $3.4 \pm 3.7, p<0.05$; HF-sBP: $1.8 \pm 1.8$ vs. $1.0 \pm 0.7, p<0.05$; LF-dBP: $5.9 \pm 4.7$ vs. $3.5 \pm 3.2, p<0.05)$ more than SD. Post hoc analysis revealed that after SD mean value of CBT from 21:30 to 06:30 was significantly higher compared to normal night's sleep and SF. In healthy men SF affects the hemodynamic and autonomic changes more than SD. Sympathetic overactivity is the proposed underlying mechanism.
\end{abstract}

\section{Key words}

Autonomic nervous system • Circadian • Adaptation

\section{Corresponding author}

P. Zalewski, Ludwik Rydygier Collegium Medicum in Bydgoszcz, Nicolaus Copernicus University in Torun, Department of Hygiene,
Epidemiology and Ergonomics, ul. M. Sklodowskiej-Curie 9, 85-094 Bydgoszcz, Poland. E-mail: p.zalewski@cm.umk.pl

\section{Introduction}

Sleep loss and sleep deprivation have been studied for the past century and are known to have negative effects on metabolism, cognitive and neurobehavioral functions, inflammatory system and cardiovascular regulation. Epidemiological studies report an increased risk of cardiovascular morbidity and mortality among persons who report short or long sleep duration. Recent studies have revealed relationships between sleep deprivation and coronary heart disease, hypertension and diabetes mellitus (Thomas and Calhoun 2016, Morris et al. 2012, Palma et al. 2013). Numerous studies describe the effect of shift work on disruption of the circadian rhythm of core body temperature (CBT) (Krauchi and Wirz-Justice 2001, Krauchi and Wirz Justice 1994). All of these studies have primarily focused on duration of nighttime sleep and have not independently considered the potential risk associated with napping (Gangwish 2014, Faraut et al. 2016).

Unlike sleep deprivation, the relationship between sleep fragmentation/napping and CBT is less clear. In this study we set out to understand is sleep fragmentation affects the cardiovascular regulation more or less than sleep deprivation. Therefore the aim of this study was to analyze dynamic fluctuations in the 
circadian rhythm of CBT in healthy adults exposed to experimental sleep deprivation compared to sleep fragmentation, using fully objective measurement methods.

\section{Methods}

\section{Subjects}

The study included volunteers, healthy, adult men, aged 20-40 years old. Apart from giving their voluntary consent to participation in the study, the main enrolment criteria included sex, no co-morbidity, no reported sleep disorders (Pittsburgh Sleep Quality Index $<5$ ). The exclusion criteria were: shift work in the past 2 years, drinking more than two cups of caffeinated drinks or two standard drinks of alcohol per day, sport at competitive level, BMI above $30 \mathrm{~kg} / \mathrm{m}^{2}$, taking any medicines / supplements during the study, cardiovascular disorders observed during the study. Clinical assessment of subjects included: a basic examination, and evaluation of the autonomic nervous system (shortened Low's questionnaire) performed by a doctor.

A permit from the Bioethics Commission of the Collegium Medicum in Bydgoszcz of Nicolaus Copernicus University in Torun to carry out this study was obtained.
Design

The entire study period was 2 nights. During the experiment the subjects stayed in the chronobiology laboratory (windowless and sound-insulated room, temperature $22{ }^{\circ} \mathrm{C}$, humidity $60 \%$, light bedcover) employing a constant routine. Subjects reported to the laboratory in the evening for an 8-h sleep adaptation episode. Additionally, the device Actigraph GT3X was used during the adaptive night - total sleep time was $\mathrm{TST}=421.2 \pm 68.2 \mathrm{~min}$, sleep efficiency $\mathrm{SE}=95.5 \pm 3.0$ and wake after sleep onset $\mathrm{WASO}=18.1 \pm 12.2$.

They ate the same meals at the same time of the day. Water $(100 \mathrm{ml})$ was administered at hourly intervals. They were cared for by trained personnel for $24 \mathrm{~h}$.

Subjects were randomized to one of two groups: group A (sleep deprivation) and group B (sleep fragmentation), Figure 1. After the adaptive night physical activity was restricted to a minimum, subject were not allowed to drink caffeine containing liquids. On the second night subjects from group B remained in bed fropm 22:00 till 9:00 (semirecumbent during wakefulness and supine during scheduled sleep episodes). 3 alternating sleep-wake cycles (or nap cycles, naps 1-3) of 150 min of scheduled wakefulness (light phase, $<8$ lux) and of $75 \mathrm{~min}$ of scheduled sleep (dark phase, 0 lux). The low-light intensity ( $<8$ lux) was chosen because it is below the threshold for suppressing melatonin secretion.

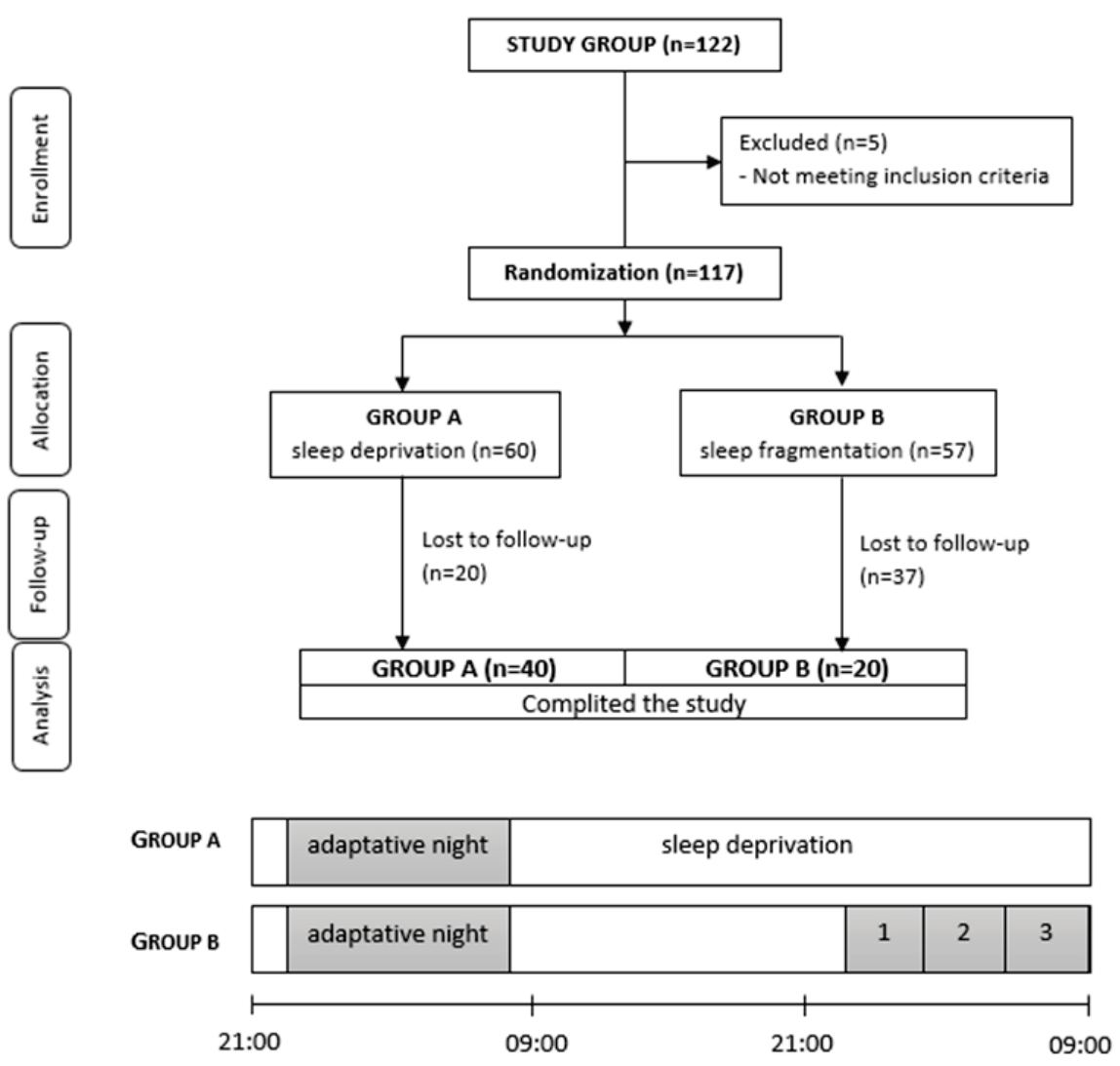

Fig. 1. Study protocol. 


\section{Measurements}

Functional assessment of the autonomic nervous system was carried out in a non-invasive manner, using the Task Force Monitor (TFM) system (model 3040i by CNSystems Medizintechnik, Graz, Austria). The main area of TFM application is as an automated and computerized beat-to-beat analysis of impedance cardiography (ICG), electrocardiogram (ECG), oscillometric and non-invasive continuous blood pressure measurement (oscBP, contBP). The evaluation included hemodynamic parameters, parameters of myocardial contractility, parameters of the spectral analysis of heart rate and blood pressure variability: HRV and BPV, parameters of the sensitivity of arterial baroreflex function. All the functions of the Task Force Monitor have been validated prior to the study, and the instrument has already been used successfully in numerous advanced clinical and scientific projects (Fortin et al. 1998, Fortin et al. 2006).

Measurements of cardiovascular system parameters took place twice: 9:00 (baseline, after adaptive night) on the first day of the study and also at 9:00 after $24 \mathrm{~h}$ of sleep deprivation (group A) or after sleep fragmentation (group B).

Core body temperature (CBT) was measured with a telemetric system Vital Sense from Mini Mitter, currently Philiphs Respironics (Vital Sense, Mini Mitter Co. Inc., Bend Oregon, USA). The system consists of two components: a mobile recording display storing and exporting digital data for measured temperature values, and a telemetric capsule - Core Body Temperature Capsule (CBTC). The telemetric capsule transmits the measured core body temperature values by radio (McKenzie and Osgood 2004). For a detailed analysis of dynamics of the core temperature fluctuations and to avoid errors resulting from possible single and occasional artefacts appearing during temperature measurements, a specific form of analysis of core temperature measurements was applied. Signals obtained throughout the study were divided into 20-minute measurement intervals, for which core temperature means were calculated and then analysed statistically.

\section{Statistical methods}

All data are presented as means \pm SD. Normal distribution of the study variables was verified with the Shapiro-Wilk test. Levane's test was used to check the homogeneity of variances in the analyzed samples. To analyze differences in results among several groups (depending on protocol), the non-parametric ANOVA Kruskal-Wallis test was used. For the detailed comparative analysis of results among separate groups the post hoc testing for multiple comparisons was used. All calculations were performed with the package Statistica 10 (StatSoft), with the assumed level of statistical significance of $\alpha<0.05$.

\section{Results}

We initially recruited 52 healthy men volunteers for the study. 2 subjects were excluded before study entry because of elevated blood pressure. 5 subjects were excluded from the final analysis because they did not comply with the study design schedule. We therefore included in the final analysis 45 subjects (mean: age $29.0 \pm 3.1$ years; height $1.79 \pm 0.1 \mathrm{~m}$, weight $80.4 \pm 9.9 \mathrm{~kg}$; BMI $24.3 \pm 2.1 \mathrm{~kg} / \mathrm{m}^{2}$ ). Table 1 shows the mean results for cardiovascular and autonomic parameters before and after sleep deprivation and sleep fragmentation.

Mean values of core body temperature during adaptive night, 24-h sleep deprivation (group A) and sleep fragmentation (group B) are shown graphically in Figure 2. There were no significant differences between CBT over $24 \mathrm{~h}$ in normal sleepers (adaptive night) compared to those undergoing sleep fragmentation. Post hoc analysis revealed that in group B mean value of core body temperature from $21: 30$ to 06:30 was significantly higher compared to group A and group $\mathrm{C}$ $(\mathrm{p}<0.01)$.

\section{Discussion}

The major finding in this study is that sleep fragmentation affects the hemodynamics, notably stroke index (SI) with significant changes in the activity of the autonomic nervous system more than sleep deprivation.

Sleep fragmentation is defined by the presence of arousals characterized by central nervous system reactivity that causes changes in cardiovascular parameters, such as RR intervals (RR), blood pressure (BP), and systemic vascular resistance, under autonomic control. The differences in activity of the autonomic nervous system detected in this study are in keeping with previous reports that confirm that sleep fragmentation is associated with sympathetic nervous system activation, elevated systolic BP and higher risk of hypertension, after controlling for confounders (Dettoni et al. 2012). Chouchou et al. (2013) shows that sleep fragmentation and indices of sympathetic activation were associated with elevated systolic BP and higher risk of systolic hypertension in a large population of elderly volunteers. 
This result was independent of the influence of SDB, hypoxaemic load, sex, BMI, diabetes, hypercholesterolaemia, and self-reported sleep duration and quality (Chouchou et al. 2013). Our previous study showed that sleep deprivation during night work evoked changes in circadian blood pressure curve during next $24 \mathrm{~h}$, increasing blood pressure during day- and night time. This effect was especially explicit in subjects of morning chronotype. Increase in blood pressure was related to the decreased baroreceptor sensitivity and their impaired circadian rhythmicity (unpublished results). Clinical effects of sleep fragmentation versus sleep deprivation on cardiovascular regulation are less known. Recent studies prove that common symptoms associated with sleep fragmentation and sleep deprivation include increased objective sleepiness, decreased psychomotor performance on a number of tasks including tasks involving short term memory, reaction time, or vigilance; and degraded mood. Both sleep fragmentation and sleep deprivation can exacerbate sleep pathology by increasing the length and pathophysiology of sleep apnea. There are many instances of sleep fragmentation as a component in both medical illnesses (fibrosis, intensive-care-unit syndrome, chronic pain and movements disorders) and liferequirements (infant care, medical residents, shift work). Most of these situations are a combination of chronic partial sleep loss and chronic sleep fragmentation. NREM sleep is characterized by marked stability of autonomic regulation with a high degree of parasympathetic neural tone, prominent respiratory sinus arrhythmia. Baroreceptor gain is high and contributes to the stability of arterial blood pressure. During REM sleep sympathetic activity increases and is concentrated in irregular periods. Heart rate and blood pressure reach levels higher than during wakefulness, with increased variability. Sleep fragmentation is probably related more to the shortening of NREM sleep (than REM sleep). Thus, during night higher level of sympathetic activity is stabilized, resulting in fixation of this pattern. Sleep deprivation does not promote an increase in sympathetic activity, typical for REM sleep (Bonnet and Arand 1997, Sommers et al. 1993, Mancia 1993, Smyth et al. 1969).

Table 1. Mean values for cardiovascular and autonomic parameters before (01) and after (02) sleep deprivation and sleep fragmentation.

\begin{tabular}{|c|c|c|c|c|c|c|}
\hline Parameter & $\begin{array}{c}\text { Sleep } \\
\text { deprivation } 01\end{array}$ & $\begin{array}{c}\text { Sleep } \\
\text { fragmentation } 01\end{array}$ & $\mathbf{p}$ & $\begin{array}{c}\text { Sleep } \\
\text { deprivation } 02\end{array}$ & $\begin{array}{c}\text { Sleep } \\
\text { fragmentation } 02\end{array}$ & $\mathbf{p}$ \\
\hline \multicolumn{7}{|c|}{ Hemodynamic parameters } \\
\hline$H R$ & $57.8 \pm 7.5$ & $63.3 \pm 5.6$ & 0.0014 & $56.2 \pm 7.6$ & $61.9 \pm 5.6$ & 0.0055 \\
\hline$s B P$ & $124.0 \pm 9.4$ & $124.8 \pm 8.9$ & $\mathrm{p}>0.05$ & $121.1 \pm 7.6$ & $123.8 \pm 7.3$ & $\mathrm{p}>0.05$ \\
\hline$d B P$ & $79.7 \pm 7.6$ & $79.7 \pm 7.4$ & $\mathrm{p}>0.05$ & $76.3 \pm 5.8$ & $79.6 \pm 6.2$ & $\mathrm{p}>0.05$ \\
\hline$m B P$ & $96.4 \pm 8.3$ & $93.4 \pm 8.5$ & $\mathrm{p}>0.05$ & $93.7 \pm 5.9$ & $92.7 \pm 6.3$ & $\mathrm{p}>0.05$ \\
\hline$S I\left(m l / m^{2}\right)$ & $57.0 \pm 13.1$ & $56.1 \pm 12.4$ & $\mathrm{p}>0.05$ & $59.8 \pm 12.2$ & $52.7 \pm 11.1$ & 0.0391 \\
\hline$C I\left(l / \mathrm{min} / \mathrm{m}^{2}\right)$ & $3.3 \pm 0.8$ & $3.6 \pm 0.9$ & $\mathrm{p}>0.05$ & $3.4 \pm 0.8$ & $3.2 \pm 0.8$ & $\mathrm{p}>0.05$ \\
\hline $\operatorname{TPRI}\left(d y n *{ }^{*} * m^{2} / \mathrm{cm}^{5}\right)$ & $2,456.6 \pm 775$ & $2,215.6 \pm 749.4$ & $\mathrm{p}>0.05$ & $2,316.3 \pm 655.6$ & $2,371.7 \pm 652.9$ & $\mathrm{p}>0.05$ \\
\hline \multicolumn{7}{|c|}{ Spectral analysis of $H R V$} \\
\hline$L F\left(m s^{2}\right)$ & $1,299.0 \pm 1,665.4$ & $843.2 \pm 66.9$ & $\mathrm{p}>0.05$ & $1,317.3 \pm 1,486.9$ & $1,388.9 \pm 963.1$ & $\mathrm{p}>0.05$ \\
\hline$H F\left(m s^{2}\right)$ & $1,496.0 \pm 3,261.1$ & $610.2 \pm 489.8$ & $\mathrm{p}>0.05$ & $1,630.2 \pm 4,415.6$ & $1,034.3 \pm 1,004.9$ & $\mathrm{p}>0.05$ \\
\hline \multicolumn{7}{|c|}{ Spectral analysis of BPV } \\
\hline$L F-s B P\left(m m H g^{2}\right)$ & $4.1 \pm 6.2$ & $5.9 \pm 5.5$ & $\mathrm{p}>0.05$ & $3.4 \pm 3.7$ & $6.0 \pm 5.3$ & 0.0367 \\
\hline$H F-s B P\left(m m H g^{2}\right)$ & $1.3 \pm 1.5$ & $1.6 \pm 1.3$ & $\mathrm{p}>0.05$ & $1.0 \pm 0.7$ & $1.8 \pm 1.8$ & 0.0255 \\
\hline$L F-d B P\left(m m H g^{2}\right)$ & $3.6 \pm 2.7$ & $5.5 \pm 4.7$ & $\mathrm{p}>0.05$ & $3.5 \pm 3.2$ & $5.9 \pm 4.7$ & 0.0336 \\
\hline$H F-d B P\left(m m H g^{2}\right)$ & $0.8 \pm 0.6$ & $0.7 \pm 0.4$ & $\mathrm{p}>0.05$ & $0.8 \pm 0.9$ & $1.1 \pm 0.5$ & $\mathrm{p}>0.05$ \\
\hline \multicolumn{7}{|l|}{ Baroreflex sensitivity } \\
\hline $\begin{array}{l}\text { Total-Events Slope } \\
(\mathrm{ms} / \mathrm{mm} \mathrm{Hg})\end{array}$ & 0 & $21.4 \pm 8.5$ & $\mathrm{p}>0.05$ & $27.4 \pm 13.1$ & $24.8 \pm 12.4$ & $\mathrm{p}>0.05$ \\
\hline
\end{tabular}

$\mathrm{HR}$ - heart rate, sBP - systolic blood pressure, dBP - diastolic blood pressure, mBP - mean blood pressure, SI - stroke index, CI cardiac index, TPRI - total peripheral resistance index, HRV - heart rate variability, BPV - blood pressure variability, LF - low frequency, $\mathrm{HF}$ - high frequency. 


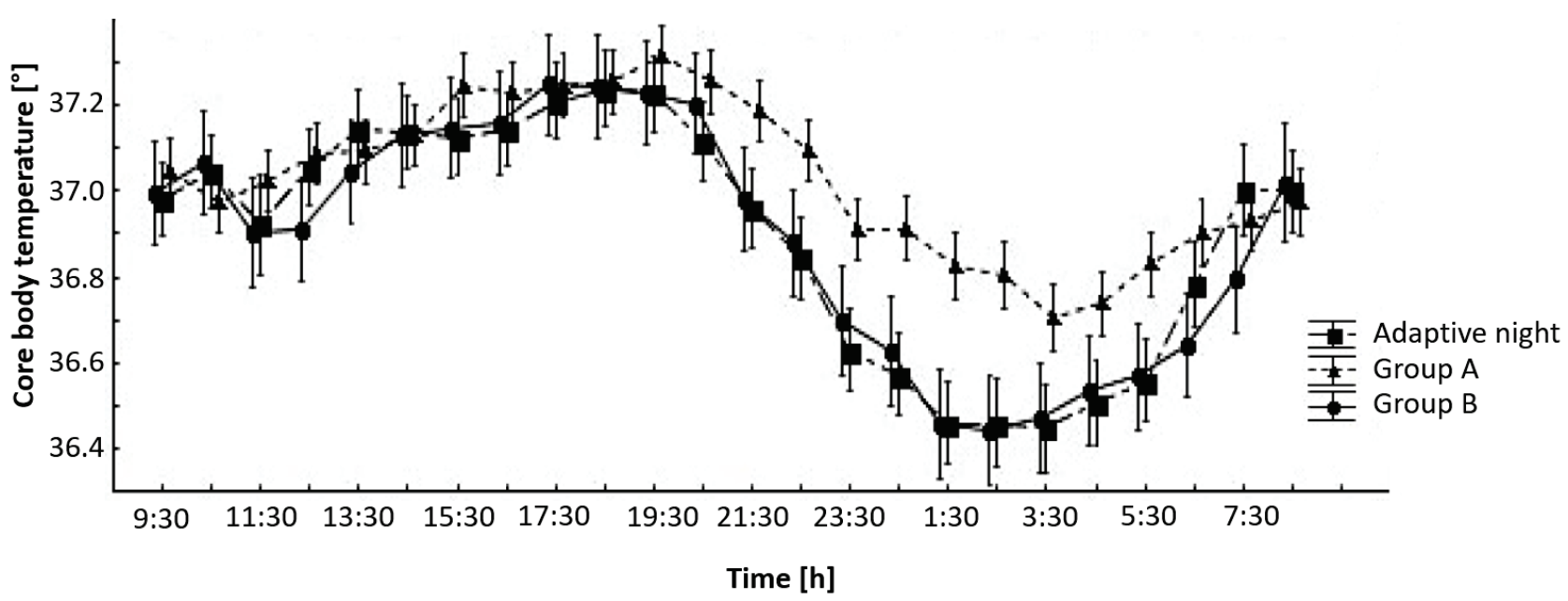

Fig. 2. Circadian fluctuations in the core body (group A - sleep deprivation, group B - sleep fragmentation).

Interestingly, this study has confirmed that there are no differences in CBT circadian rhythm after adaptive night and those undergoing a sleep fragmentation regime. There was however a significant increase in CBT from 21:30 till 07:30 in the sleep deprivation group. These results are in keeping with previous studies. Launay et al. (2002) indicated that sleep deprivation causes a significant increase in a minimum temperature, from $36.1{ }^{\circ} \mathrm{C}$ before the experiment to $36.5^{\circ} \mathrm{C}$ after $62 \mathrm{~h}$ of sleep deprivation (Launay 2002). Similar results obtained by Murray et al. (1958) indicate that during 98-hours of sleep deprivation core body temperature maintains its sinusoidal pattern with accompanying gradual reduction in its amplitude (Murray 1958, Kelly 2007). Vaara et al. (2009) suggest that this phenomenon may result from a direct effect of sleep deprivation on reduced activity of cerebral centers, including the hypothalamus, disrupting the circadian rhythm of the core body temperature. Numerous studies describe the effect of shift work on disruption of the circadian rhythm of the core body temperature. Findings in people not tolerating shift work included reduction in the circadian fluctuations in the core body temperature, a shift in the daily maximum, and appearance of free rhythms of the frequency other than 24 h (Vaara 2009, Gupta and Pati 1994, Pati and Saini 1991).

The available literature confirms that the measurement method used to measure the core body temperature influences results (Krauchi 2002). Many authors consider blood temperature in the pulmonary artery as the correct core body temperature. The most common, due to its easy availability, is measurement of the body temperature in the axilla and measurement with an infra-red sensor placed near the tympanic membrane.
An important shortcoming of these measurement methods is the fact that the temperature of the tympanic membrane or the axilla frequently differs significantly on both sides and between successive measurements. Rectal measurement is considered accurate, but relatively rarely used in experiments, and it is significantly correlated with temperature measurements conducted in the pulmonary artery. One of the relatively recently developed methods for measurement of core and surface body temperature is the use of remote temperature sensors, transmitting measured values by radio. The use of a telemetric capsule and a dermal (skin) sensor was first described in 1968. A robust development of digital technologies has allowed development of this easily available, non-invasive and very reliable method for temperature measurements (Fulbrook 1997). Advantages of this method include: the ability to obtain continuous core temperature measurements, observations of circadian dynamic fluctuations in the core body temperature and measurement precision, together with the repeatability and reliability of results (Byrne and Lim 2007, Lim et al. 2008). Studies indicate that there is a strong correlation between measurements of the core body temperature with the Vital Sense system and blood temperature in the pulmonary artery $(\mathrm{r}=0.96, \mathrm{p}<0.0001)$ (Giuliano 1999).

These findings have implications. Sleep fragmentation affects the hemodynamics with significant changes in the activity of the autonomic nervous system more than sleep deprivation. It would therefore be anticipated that the adverse consequences of sleep deprivation such as hypertension and excess cardiovascular mortality are of more significance in those who nap compared to those who are sleep deprived. Unlike sleep deprivation, sleep fragmentation using the 
protocol outlined in this study does not appear to impact upon the circadian rhythm of CBT.

\section{Conflict of Interest}

There is no conflict of interest.

\section{Acknowledgements}

We thank to Dr. Tomasz Kowalik for supporting participants of the study during the experiment.

\section{References}

BONNET MH, ARAND DL: Heart rate variability: sleep stage, time of night, and arousal influences. Electroencephalogr Clin Neurophysiol 102: 390-396, 1997.

BYRNE CH, LIM CHL: The ingestible telemetric body core temperature sensor: a review of validity and exercise applications. Br J Sports Med 41: 126-133, 2007.

CHOUCHOU F, PICHOT V, PEPIN JL, TAMISIER R, CELLE S, MAUDOUX D, GARCIN A, LEVY P, BARTHELEMY JC, ROCHE F, PROOF STUDY GROUP: Sympathetic overactivity due to sleep fragmentation is associated with elevated diurnal systolic blood pressure in healthy elderly subjects: the PROOF-SYNAPSE study. Eur Heart J 34: 2122-2131, 2131a, 2013.

DETTONI JL, CONSOLIM-COLOMBO FM, DRAGER LF, RUBIRA MC, SOUZA SB, IRIGOYEN MC, MOSTARDA C, BORILE S, KRIEGER EM, MORENO H, LORENZI-FILHO G: Cardiovascular effects of partial sleep deprivation in healthy volunteers. $J$ Appl Physiol 113: 232-236, 2012.

FARAUT B, ANDRILLON T, VECCHIERINI MF, LEGER D: Napping: A public health issue. From epidemiological to laboratory studies. Sleep Med Rev 35: 85-100, 2017.

FORTIN J, KLINGER T, WAGNER CH, STERNER H, MADRITSCH CH, GRULLENBERGER R: The task force monitor - a non-invasive beat-to-beat monitor for hemodynamic and autonomic function of the human body. Proceedings of the 20th Annual International Conference of the IEEE Engineering in Medicine and Biology Society, 1998, Hong Kong.

FORTIN J, MARTE W, GRULLENBERG R: Continuous non-invasive blood pressure monitoring using concentrically interlocking control loops. Comput Biol Med 36: 941-957, 2006.

FULBROOK P: Core body temperature measurement: a comparison of axilla, tympanic membrane and pulmonary artery blood temperature. Intensive Crit Care Nurs 13: 266-272, 1997.

GANGWISCH JE: A review of evidence for the link between sleep duration and hypertension. Am J Hypertens 27: 1235-1242, 2014.

GIULIANO KK, SCOTT SS, ELLIOT S, GIULIANO AJ: Temperature measurement in critically ill orally intubated adults: a comparison of pulmonary artery core, tympanic, and oral methods. Crit Care Med 27: 2188-2193, 1999.

GUPTA S, PATI AK: Desynchronization of circadian rhythms in a group of shift working nurses: effects of pattern of shift rotation. J Hum Ergol (Tokyo) 23: 121-131, 1994.

KELLY GS: Body temperature variability (Part 2): masking influences of body temperature variability and a review of body temperature variability in disease. Altern Med Rev 12: 49-62, 2007.

KRAUCHI K: How is the circadian rhythm of core body temperature regulated? Clin Auton Res 12: 147-149, 2002.

KRAUCHI K, WIRZ-JUSTICE A: Circadian clues to sleep onset mechanisms. Neuropsychopharmacology 25 (5 Suppl): S92-S96, 2001.

KRAUCHI K, WIRZ-JUSTICE A: Circadian rhythm of heat production, heart rate, and skin and core temperature under unmasking conditions in men. Am J Physiol 267: R819-R829, 1994.

LAUNAY JC, SAVOUREY G, GUINET A, LALLEMENT G, BESNARD Y, BITTEL J: Circadian rhythm of rectal temperature during sleep deprivation with modafinil. Aviat Space Environ Med 73: 985-990, 2002.

LIM CHL, BYRNE CH, LEE JKW: Human thermoregulation and measurement of body temperature in exercise and clinical settings. Ann Acad Med Singapore 37: 347-353, 2008.

MANCIA G: Autonomic modulation of the cardiovascular system during sleep. $N$ Engl J Med 328: 347-349, 1993. 
MCKENZIE JE, OSGOOD DW: Validation of a new telemetric core temperature monitor. J Therm Biol 29: 605-611, 2004.

MORRIS CHJ, YANG JN, SCHEER JL: The impact of the circadian timing system on cardiovascular and metabolic function. Prog Brain Res 199: 337-358, 2012.

MURRAY EJ, WILLIAMS HL, LUBIN A: Body temperature and psychological ratings during sleep deprivation. $J$ Exp Psychol 56: 271-273, 1958.

PALMA JA, URRENSTARAZU E, IRIARTE J: Sleep loss as a risk factor of neurologic disorders: A review. Sleep Med 14: 229-236, 2013.

PATI AK, SAINI SK: Desynchronization of oral temperature, pulse and performance circadian rhythms in shift working Indian nurses. Indian J Exp Biol 29: 1017-1021, 1991.

SMYTH HS, SLEIGHT P, PICKERING GW: Reflex regulation of arterial pressure during sleep in man. A quantitative method of assessing baroreflex sensitivity. Circ Res 24: 109-121, 1969.

THOMAS SJ, CALHOUN D: Sleep, insomnia, and hypertension: current findings and future directions. J Am Soc Hypertens 11: 122-129, 2017.

VAARA J, KYROLAINEN H, KOIVU M, TULPPO M, FINNI T: The effect of 60-h sleep deprivation on cardiovascular regulation and body temperature. Eur J Appl Physiol 105: 439-444, 2009. 